\title{
Characterization of chicken splenic-derived dendritic cells following vaccine and very virulent strains of infectious bursal disease virus infection
}

\begin{abstract}
Studies have shown that infectious bursal disease virus (IBDV) infects lymphoid cells, mainly B cells and macrophages. This study was aimed to examine the involvement of chicken splenic-derived dendritic cells (ch-sDCs) in specific-pathogen-free chickens following inoculation with IBDV vaccine strain (D78) and a very virulent (vv) strain (UPM0081). Following IBDV infection, enriched activated ch-sDCs were collected by using the negative selection method and were examined based on morphology and immunophenotyping to confirm the isolation method for dendritic cells (DCs). The presence of IBDV on enriched activated ch-sDCs was analyzed based on the immunofluorescence antibody test (IFAT), flow cytometry, and quantitative real-time PCR (RT-qPCR) while the mRNAs of several cytokines were detected using RT-qPCR. The isolated ch-sDCs resembled typical DC morphologies found in mammals by having a veiled shape and they grew in clusters. Meanwhile, the expression of DC maturation markers, namely CD86 and MHCII, were increased at day 2 and day 3 following vvIBDV and vaccine strain inoculation, respectively, ranging from $10 \%$ to $40 \%$ compared to the control at $2.55 \%(P<0.05)$. At day 3 postinfection, IBDV VP3 proteins colocalized with CD86 were readily detected via IFAT and flow cytometry in both vaccine and vvIBDV strains. In addition, enriched activated chsDCs were also detected as positive based on the VP4 gene by RT-qPCR; however, a higher viral load was detected on vvIBDV compared to the vaccine group. Infection with vaccine and vvIBDV strains induced the enriched activated ch-sDCs to produce proinflammatory cytokines and Th1-like cytokines from day 3 onward; however, the expressions were higher in the vvIBDV group $(P<0.05)$. These data collectively suggest that enriched activated chsDCs were permissive to IBDV infection and produced a strong inflammatory and Th1-like cytokine response following vvIBDV infection as compared to the vaccine strain.
\end{abstract}

Keyword: Chicken splenic-derived dendritic cells (ch-sDCs); Infectious bursal disease virus (IBDV); Vaccine strain; Very virulent IBDV (vvIBDV); Cytokines 
